evidence that anxiety may influence cognitive test scores in this population. Future research should examine the influence of anxiety during post-concussion assessment when stakes may be higher in this underrepresented population.

\section{TESTING NEUROCOGNITIVE FUNCTION AND BALANCE FOLLOWING SPORT CONCUSSION - DO WE NEED BASELINES?}

1,2Michael Hutchison, ${ }^{1}$ Alex Di Battista, ${ }^{1}$ Kyla Pyndiura, ${ }^{1,2}$ Doug Richards. ${ }^{1}$ University of Toronto, Toronto, Canada; ${ }^{2}$ David L. Maclntosh Sport Medicine Clinic, Toronto, Canada

\subsection{6/bjsports-2021-IOC.159}

Background While evaluating balance and neurocognitive function following concussion is clinically valuable for both diagnosis and treatment, it is unclear if these assessments are best employed in reference to a pre-injury evaluation, or to normative results sampled from a healthy population.

Objective To evaluate the performance of a computerized assessment of balance and neurocognitive function in athletes following a sport-related concussion (SRC) compared to either their own pre-injury test scores, or the scores of a normative sample of uninjured athletes.

Hypothesis SRC test scores would discriminate better from athlete's own pre-injury scores compared to those of an uninjured cohort.

Design Prospective observational study design.

Setting Sport medicine clinic at a Canadian University.

Participants One hundred and forty Canadian Interuniversity athletes ( $\mathrm{n}=73$ male, $\mathrm{n}=67$ female) from 12 different sports. Seventy non-injured athletes $(\mathrm{n}=31$ male, $\mathrm{n}=39$ female) were assessed prior to the start of their competitive season, while 70 athletes ( $\mathrm{n}=42$ male, $\mathrm{n}=28$ female) with SRC were assessed within one week of injury.

Assessment of Risk Factors At the time of testing, fourteen measures of neurocognitive function $(\mathrm{n}=6)$ and balance ( $\mathrm{n}$ $=8$ ) were assessed using the C3 Logix application (NeuroLogix Technologies, Cleveland, Ohio).

Main Outcome Measures Presence or absence of SRC, as diagnosed by a sport medicine doctor.

Results Partial least squares discriminant analyses followed by the evaluation of model classification accuracy (Accur) revealed better discriminative performance when test scores in athletes with SRC were compared to an uninjured cohort (Accur $=0.74$ ), as opposed to their own pre-injury test scores (Accur $=0.61$ ).

Conclusions Contrary to our hypothesis, these initial results suggest that neurocognitive and balance testing following SRC may benefit from comparison to normative reference values sampled from a healthy population.

\section{DO FUTURE DOCTORS IN SCOTLAND NEED FURTHER EDUCATION TO ENSURE THEY CAN ADEQUATELY IDENTIFY AND MANAGE CONCUSSIONS?}

${ }^{1}$ Christopher Jervis, ${ }^{2}$ Stephanie Adams, ${ }^{2}$ Samantha Fawkner, ${ }^{2,3,4}$ Steffan Griffin. ${ }^{1}$ The University of Edinburgh Medical School, 47 Little France Crescent, Edinburgh, EH16 4TJ, Edinburgh, UK; ${ }^{2}$ Moray House School of Education and Sport, The University of Edinburgh, Holyrood Road, Edinburgh, EH8 8AQ, Edinburgh, UK; ${ }^{3}$ Centre for Sport and Exercise, The University of Edinburgh, Edinburgh, UK., Edinburgh, UK; ${ }^{4}$ Medical Services, Rugby Football Union, London, UK., London, UK

10.1136/bjsports-2021-IOC. 160
Background Preventing sports-related concussions is a medical priority across all levels of contact sports. Doctors play a key role in ensuring concussed athletes can safely return to sport, yet research has shown deficiencies in concussion knowledge and education amongst doctors and medical students. In the UK, studies assessing concussion knowledge and learning experiences have not previously been conducted in medical schools despite a parliamentary inquiry highlighting evidence regarding the links between sport and brain injury.

Objective To assess medical students' concussion knowledge and learning needs in Scottish Medical Schools.

Design An online survey with 23 questions assessing aspects of concussion prevention, detection and management was distributed to Scottish Medical schools. Data collection took place between October and December 2020. Scores were calculated based on correct recognition of symptom and management approaches.

Setting Clinical year medical students studying in Scotland.

Participants 200 medical students completed the survey $(8 \%$ response rate).

Results On average, participants scored $87.3 \%$ (sd 9.56) on symptom recognition items but only $31 \%$ (sd 15.3) on questions relating to management and prevention. $38 \%$ incorrectly identified that brain imaging could diagnose a concussion. Only $15 \%$ of participants correctly identified that headgear does not prevent concussions and only one participant correctly identified the minimum 'return to sport' timeframes for adults and children. 15\% of participants reported having learnt about concussion at medical school and 92.5\% were interested in receiving concussion teaching at medical school.

Conclusions Medical students show competence in concussion recognition but there are gaps in knowledge concerning concussion prevention and management. Concussion is not frequently taught in medical schools despite a desire for it to be covered in curricula. Further studies are needed to understand how concussion education can best be incorporated to ensure future doctors are competent in managing concussions and minimising the risk of secondary harms.

\section{PREVENTION AND MANAGEMENT OF SPORT-RELATED CONCUSSIONS: CURRENT KNOWLEDGE AND PRACTICES AMONG FIELD STAKEHOLDERS OF THE FRENCH- SPEAKING IOC RESEARCH CENTRE (REFORM)}

${ }^{1}$ Géraldine Martens, ${ }^{1}$ Jean-François Kaux, ${ }^{6}$ Aurore Thibaut, ${ }^{2}$ Philippe Tscholl, ${ }^{3}$ Axel Urhausen, ${ }^{4}$ Sébastien Le Garrec, ${ }^{5}$ Suzanne Leclerc. ${ }^{1}$ Réseau Francophone Olympique de la Recherche en Médecine du Sport (ReFORM), Centre Hospitalier Universitaire de Liège, Liège, Belgium; ${ }^{2}$ Réseau Francophone Olympique de la Recherche en Médecine du Sport (ReFORM), Hôpitaux Universitaires de Genève, Genève, Switzerland; ${ }^{3}$ Réseau Francophone Olympique de la Recherche en Médecine du Sport (ReFORM), Centre Hospitalier de Luxembourg, Luxembourg, Luxembourg; ${ }^{4}$ Réseau Francophone Olympique de la Recherche en Médecine du Sport (ReFORM), Institut National du Sport, de I'Expertise et de la Performance, Paris, France; ${ }^{5}$ Réseau Francophone Olympique de la Recherche en Médecine du Sport (ReFORM), Institut National du Sport du Québec, Montréal, Canada; ${ }^{6}$ Coma Science Group - GIGA Consciousness, Centre Hospitalier Universitaire de Liège, Liège, Belgium

\subsection{6/bjsports-2021-IOC.161}

Background Sport-related concussions (SRC) impact the practice of numerous athletes. Their appropriate management involves education and prevention among all field stakeholders including physicians, coaches and athletes themselves. 
Objective To assess the current state of knowledge and practice regarding SRC (diagnosis, treatment, return to play) within the French-speaking sports community in order to tailor the prevention approaches.

Design Multicentric cross sectional survey.

Setting An online survey ( 15 minutes completion time) was sent through mailing lists and social networks. The survey was available for three months and monthly reminders were sent.

Patients (or Participants) Athletes, sports healthcare professionals, and coaches through the ReFORM network

Interventions (or Assessment of Risk Factors) N/A

Main Outcome Measurements Reported level of knowledge regarding SRC and access to educational resources.

Results 2072 participants took part in the survey and 1704 finished it (completion rate: $82 \%$ ). The sample included $48 \%$ of athletes, $33 \%$ of coaches and 19\% of healthcare professionals. The main countries represented were France (35\%), Canada (32\%) and Belgium (12\%). The preliminary analyses reported a SRC knowledge self-assessment as 'good' or 'excellent' in $87 \%$ of healthcare professionals and $69 \%$ of coaches; while more than $40 \%$ of athletes rated their knowledge as 'poor' or 'none'. Only 17\% of athletes reported knowing about a SRC education programme in their setting against $63 \%$ for healthcare professionals and $45 \%$ for coaches. Regarding coaches, $54 \%$ do not feel having sufficient professional resources to correctly manage a SRC over the return to sports continuum.

Conclusions There seems to be a great interest from field stakeholders reflected by the completion rate. These preliminary results show a discrepancy in the level of SRC knowledge and the access to educational resources between athletes, coaches and healthcare professionals.

\section{DO CERVICAL SPINE, VESTIBULO-OCULAR, DYNAMIC BALANCE, AND DIVIDED ATTENTION MEASURES IN ELITE YOUTH ICE HOCKEY PLAYERS RETURN TO BASELINE LEVELS AT TIME OF MEDICAL CLEARANCE TO RETURN TO PLAY?}

1,2,3,4,5 Kathryn Schneider, ${ }^{5,6}$ Geoff Schneider, ${ }^{1,2,3,7}$ Carolyn Emery. ${ }^{~}$ Sport Injury Prevention Research Centre, Faculty of Kinesiology, University of Calgary, Calgary, Canada; ${ }^{2}$ Alberta Children's Hospital Research Institute, for Child and Maternal Health, Faculty of Medicine, University of Calgary, Calgary, Canada; ${ }^{3}$ Hotchkiss Brain Institute, University of Calgary, Calgary, Canada; ${ }^{4}$ Sport Medicine Centre, University of Calgary, Calgary, Canada; ${ }^{5}$ Evidence Sport and Spinal Therapy, Calgary, Canada; ${ }^{6}$ Department of Radiology, Cummings School of Medicine, University of Calgary, Calgary, Canada; 'Departments of Pediatrics and Community Health Sciences, Cumming School of Medicine, University of Calgary, Calgary, Canada

\subsection{6/bjsports-2021-IOC. 162}

Background Evaluation of multiple sensory and motor domains at time of return to play (RTP) may inform risk of recurrent concussion and injury following concussion.

Objective To evaluate if measures of (1) cervical spine function, (2) vestibulo-ocular reflex (VOR) function, (3) dynamic balance and (4) tasks of divided attention have returned to preinjury levels at medical clearance to RTP in elite youth ice hockey players.

Design Case series nested in a prospective cohort study $(\mathrm{n}=559)$.

Setting Canadian youth ice hockey.

Participants Youth ice hockey players [13-17 years; $n=45$ (8 female, 37 male)].
Interventions Players who were diagnosed with an ice hockey-related concussion completed preseason and RTP measures.

Main Outcome Measurements Cervical spine measures (cervical flexor endurance test, head perturbation test, anterolateral strength, cervical flexion rotation test, joint position error), VOR tests [head thrust test, dynamic visual acuity (clinical and computerized)], dynamic balance tests (functional gait) and divided attention tasks (walking-while-talking-test WWTT) were included. Non-parametric (Wicoxon signed-rank, Stuart-Maxwell) analyses compared preseason to RTP scores.

Results Symptoms of dizziness, neck pain, and headache were reported by 29\%, 18\% and 20\% fewer players at RTP than preseason respectively. Anterolateral cervical muscle strength $(\mathrm{z}=-5.16, \mathrm{p}<0.0001)$ and joint position error (left) $(\mathrm{z}=2.91$, $\mathrm{p}=0.0036)$ were poorer at RTP compared to preseason. The WWTT time $(\mathrm{z}=-2.66, \mathrm{p}=0.0079)$ and FGA scores were improved at RTP $(\mathrm{z}=-2.55, \mathrm{p}=0.011)$.

Conclusions Anterolateral cervical spine strength and joint position error (left) did not return to preseason values at RTP and may suggest incomplete recovery not indicated by symptoms at RTP. WWTT and dynamic balance were improved at RTP. Further evaluation of clinical outcomes on risk of subsequent injury following concussion is warranted.

\section{PRIMARY PREVENTION OF SPORT-RELATED CONCUSSION IN YOUTH ICE HOCKEY: A PILOT RANDOMIZED CONTROLLED TRIAL}

\begin{abstract}
1,2,3,4,5Kathryn Schneider, ${ }^{1,2,3}$ Paul Eliason, ${ }^{1}$ Cody van Rassel, ${ }^{1,4}$ Stacy Sick, ${ }^{1}$ Lauren Miurz, ${ }^{5,6} \mathrm{Geoff}$ Schneider, ${ }^{1,2,3}$ Carolyn Emery. ${ }^{1}$ Sport Injury Prevention Research Centre, Faculty of Kinesiology, University of Calgary, Calgary, Canada; ${ }^{2}$ Alberta Children's Hospital Research Institute, Calgary, Canada; ${ }^{3}$ Hotchkiss Brain Institute, University of Calgary, Calgary, Canada; ${ }^{4}$ Acute Sport Concussion Clinic, Sport Medicine Centre, Faculty of Kinesiology, University of Calgary, Calgary, Canada; ${ }^{5}$ Evidence Sport and Spinal Therapy, Calgary, Canada; ${ }^{6}$ Department of Radiology, Cumming School of Medicine, University of Calgary, Calgary, Canada
\end{abstract}

\subsection{6/bjsports-2021-IOC.163}

Background Neuromuscular and sensorimotor training may reduce the risk of concussion.

Objective To evaluate the feasibility and efficacy of a concussion prevention neuromuscular training program in youth ice hockey.

Design Pilot cluster-randomized controlled trial.

Setting Canadian ice hockey.

Patients (or Participants) Youth ice hockey players (ages 1317).

Interventions (or Assessment of Risk Factors) A study physiotherapist visited teams weekly for six weeks. Both study groups received standard concussion education and previously validated concussion surveillance. Intervention teams completed a progressive neuromuscular and sensorimotor training program (10-20 minutes per session) (including aerobic, balance, strength, agility, adaptation, cervical spine strength and dividing attention), on- and off-ice. Control teams completed their typical warm-up.

Main Outcome Measurements Recruitment, completion, retention rates, time and safety were used to evaluate feasibility. Sport-related concussion was defined using the $5^{\text {th }}$ Consensus Statement on Concussion in Sport. 